Research in Astron. Astrophys. 2012 Vol. X No. XX, 000-000

http://www.raa-journal.org http://www.iop.org/journals/raa

\title{
Long-term optical-infrared color variability of blazars *
}

\author{
Bing-Kai Zhang ${ }^{1}$, Xiao-Shan Zhou ${ }^{1}$, Xiao-Yun Zhao ${ }^{1}$, Ben-Zhong Dai $^{2}$ \\ ${ }^{1}$ Department of Physics, Fuyang Normal College, Fuyang 236041, China; zhangbk@ihep.ac.cn \\ 2 Department of Physics, Yunnan University, Kunming 650091, China \\ Received ; accepted
}

\begin{abstract}
The long-term optical and infrared color variability of blazars has been investigated with the SMARTS monitoring data. The sample in this study consists of 49 flat spectrum radio quasars and $22 \mathrm{BL}$ Lac objects. The fractional variability amplitudes of each source have been calculated in both optical $R$ band and infrared $J$ band. Overall, the variability amplitudes of FSRQs are larger than those of BL Lac objects. The results also suggest that the variability amplitude of most FSRQs is larger at lower energy band ( $J$ band) than at higher one ( $R$ band), while the variability amplitude of BL Lacs are larger at higher energy band. Two types of blazars both display color variation along with the variability in brightness. However, they show different variation behaviors in general. With the whole data set, 35 FSRQs exhibit redder-when-brighter trends, and 11 FSRQs exhibit opposite trends; 11 BL Lacs follow bluer-when-brighter trends, and 7 BL Lacs follow opposite trends. The examination in detail shows that there are 10 blazars showing redder-when-brighter trend in their low state, and bluer-when-brighter or stable-whenbrighter trends in their high state. Some more complicated color behaviors have also been detected in several blazars. The non-thermal jet emission and the thermal emission from the accretion disc are employed to explain the observed color behaviors.
\end{abstract}

Key words: galaxies: active - BL Lacertae objects: general - quasars: general - methods: statistical

\section{INTRODUCTION}

Blazars consist of flat spectrum radio quasars (FSRQs) and BL Lacertae objects (BL Lacs). They are dominated by non-thermal continuum emission. The flux decreases along with the frequency increasing, which approximately follows a power law. Measurements of the spectral energy distribution (SEDs) play an important role in understanding the emission processes of blazars. Since blazars vary rapidly and violently at all wavelengths from radio to $\gamma$-ray with time scales from minutes to years (Carini et al. 1992; Arévalo et al. 2008; Ghosh et al. 2000; Ciprini et al. 2007; Chatterjee et al. 2012; Zhang et al. 2013; D'Ammando et al. 2013), the lack of simultaneous data at the various wavelengths is an obstacle in the broadband study of blazars. Flux variations in blazars are usually accompanied by changes in the spectral shape. Therefore, the analysis of spectral changes of blazars can put some strong constraints on the physical processes that are responsible for these variations.

The spectral change can be characterized by the color change. Color variations in blazars have been explored by several authors. For 28 radio-selected BL Lacs in Fan \& Lin (2000), two showed flatter spectra when getting brighter, while three showed opposite behavior. Vagnetti, Trevese \& Nesci (2003)

* Supported by the National Natural Science Foundation of China. 
found bluer-when-brighter (BWB) trends in the $B V R I$ bands for all 8 BL Lacs in their sample. Gu et al. (2006) found that 3 out of 5 BL Lacs exhibited significant BWB trends and 2 out of 3 FSRQs exhibited redder-when-brighter (RWB) trends. However, Gu \& Ai (2011) showed that only one FSRQs is RWB in a sample of 29 SDSS FSRQs. Rani et al. (2010) found that 3 out of 6 BL Lacs tended to become bluer with increase in brightness, and 4 out of 6 FSRQs exhibited opposite trends. Ikejiri et al. (2011) found that 28 out of 32 well observed blazars showed BWB trend. Bonning et al. (2012) found that FSRQs had redder optical-infrared colors when they were brighter, while BL Lac objects showed no such trend. Gaur et al. (2012) suggested that 6 out of 10 BL Lacs followed the BWB trend, while all 6 BL Lacs in Sandrinelli, Covino \& Treves (2014) showed no BWB trends. The color behaviors of some individual sources are also investigated in many disperse literatures (e.g. Raiteri et al. 2003; Papadakis et al. 2003; $\mathrm{Hu}$ et al. 2006; Wu et al. 2006, 2011; Dai et al. 2009; Zhang et al. 2010a; Dai et al. 2011; Fan et al. 2014).

BWB and RWB trends are two common well-observed behaviors in blazars. However, some complicated color phenomena have also been found in some cases. In general, there are five kinds of color behaviors: BWB in the whole data sets; RWB in the whole data sets; cycles or loop-like pattern; RWB at the low state while BWB at the high state (RWB to BWB); stable-when-brighter (SWB) or no correlation with brightness in the whole data sets (see Zhang et al. (2014) and references therein). Up to now, FSRQs and BL Lacs seem not to have universal features in color variations. The purpose of this paper is to investigate their color behaviors using a much larger sample of blazars with almost simultaneous measurements, and expect to provide results with higher confidence levels. The paper is organized as following: in Section 2, the infrared and optical data as well as their variations are described; in Section 3 , the color behavior is studied; then the discussion and conclusions are given in Section 4.

\section{INFRARED AND OPTICAL DATA \& VARIABILITY}

In order to investigate the statistics of the color behavior of blazars, we collect 71 blazars as well as 4 possible blazars from SMARTS sources. SMARTS program employs four meter-class telescopes (1.5m, 1.3-m, 1.0-m and 0.9-m) of the Small and Moderate Aperture Research Telescope System (SMARTS) at Cerro Tololo Interamerican Observatory (CTIO) in Chile to observe the bright southern gamma-ray blazars on a regular cadence, at both optical and near-infrared wavelengths. Data reduction and analysis of SMARTS data have been described in Bonning et al. (2012). Table 1 lists our sample which comprises 49 FSRQs, 22 BL Lacs and 4 unidentified type sources. The first column gives the object name, the second column gives the spectral classification, the third one gives the monitoring duration in days, the fourth one gives the number of data points for color index calculation. Most of the sources are observed in $B, V, R, J$ and $K$ bands. On the whole, there are the most data points in $R$ and $J$ bands. So, these two bands have been selected to study color variations. However, the observations for each source are not uniform. Some objects have been observed for more than 2000 days, while some others have been observed for less than 100 days. Some sources have been well observed with hundreds of data points and some less than 10 data points.

In order to compare the amplitude of the variations in different blazars, and in different bands for the same blazar, we compute the fractional variability amplitude $F_{\text {var }}$ (Vaughan et al. 2003), which represents the average amplitude of the observed variations as a percentage of the light curve mean. The fractional variability amplitude is defined as $F_{\text {var }}=\sqrt{\left(S^{2}-<\varepsilon_{e r r}^{2}>\right) /<x>^{2}}$, where $S^{2}$ is the sample variance of the light curve, $\langle x\rangle$ is the average flux and $\left\langle\varepsilon_{e r r}^{2}\right\rangle$ is the mean of the squared measurement uncertainties. The $F_{v a r}$ values of $J$ and $R$ bands are listed in the fifth and sixth columns of Table 1. In Fig. 1 we plot the $F_{v a r}$ of $R$ band versus that of $J$ band for each object. It can be seen that the objects show great differences in variations. Most of the objects show significant variabilities. Several sources display extreme variability, such as AO 0235-164, with $F_{v a r}=1.57$ in $J$ band and $F_{v a r}=1.59$ in $R$ band. However, there are also a few sources showing weak variabilities, such as $3 \mathrm{C} 273$, with $F_{v a r}=$ 0.06 in $J$ band and $F_{\text {var }}=0.04$ in $R$ band.

Fig. 2 plots the distribution of $F_{v a r} s$ of $R$ band and $J$ band. On the whole, $F_{v a r} s$ of $R$ band have no significant difference with those of $J$ band for all objects, which means that the variability amplitudes 
Table 1 The Property of Variations of Optical and Infrared Data.

\begin{tabular}{|c|c|c|c|c|c|c|c|c|c|c|c|c|}
\hline Object & Class & $\begin{array}{c}\Delta T \\
\text { (day) }\end{array}$ & $N$ & $J_{F v a r}$ & $R_{\text {Fvar }}$ & $r_{J-R}$ & Prob. & $r_{C-R}$ & Prob. & $r_{C-J}$ & Prob. & Behavior \\
\hline PMN 0017-0512 & FSRQ & 211 & 73 & $0.82 \pm 0.07$ & $0.67 \pm 0.06$ & 0.97 & $<10^{-9}$ & 0.67 & $<10^{-11}$ & 0.82 & $<10^{-9}$ & RWB \\
\hline PKS 0035-252 & FSRQ & 357 & 9 & $0.43 \pm 0.11$ & $0.38 \pm 0.10$ & 0.11 & 0.78 & -0.49 & 0.18 & 0.81 & $8.0 \times 10^{-3}$ & RWB \\
\hline PKS 0208-512 & FSRQ & 2016 & 381 & $0.96 \pm 0.03$ & $0.86 \pm 0.03$ & 0.96 & $<10^{-9}$ & 0.30 & $2.7 \times 10^{-9}$ & 0.55 & $<10^{-9}$ & RWB \\
\hline $4 C+01.02$ & FSRQ & 76 & 8 & $0.32 \pm 0.08$ & $0.17 \pm 0.04$ & 0.73 & 0.04 & 0.34 & 0.40 & 0.89 & $2.7 \times 10^{-3}$ & RWB \\
\hline AO $0235+164$ & FSRQ & 1816 & 221 & $1.57 \pm 0.07$ & $1.59 \pm 0.08$ & 0.98 & $<10^{-9}$ & 0.21 & $2.1 \times 10^{-3}$ & 0.38 & $4.2 \times 10^{-9}$ & RWB \\
\hline PKS 0235-618 & FSRQ & 158 & 14 & $0.25 \pm 0.05$ & $0.32 \pm 0.06$ & 0.48 & 0.09 & -0.72 & $3.9 \times 10^{-3}$ & 0.27 & 0.35 & BWB \\
\hline 1RXS 0238-3116 & BL Lac & 654 & 20 & $0.20 \pm 0.03$ & $0.29 \pm 0.05$ & -0.10 & 0.68 & -0.87 & $7.7 \times 10^{-7}$ & 0.58 & $7.2 \times 10^{-3}$ & BWB(RWB) \\
\hline PKS 0250-225 & FSRQ & 90 & 50 & $0.40 \pm 0.04$ & $0.40 \pm 0.04$ & 0.85 & $<10^{-9}$ & -0.26 & 0.07 & 0.29 & 0.04 & None \\
\hline PKS 0301-243 & BL Lac & 537 & 48 & $0.15 \pm 0.02$ & $0.18 \pm 0.02$ & 0.84 & $<10^{-9}$ & -0.56 & $4.1 \times 10^{-5}$ & -0.02 & 0.89 & BWB \\
\hline BG6 J0316+0904 & BL Lac & 783 & 12 & $0.16 \pm 0.03$ & $0.25 \pm 0.05$ & 0.92 & $2.3 \times 10^{-5}$ & -0.82 & $9.7 \times 10^{-4}$ & -0.54 & 0.07 & BWB \\
\hline PKS 0402-362 & FSRQ & 863 & 75 & $0.26 \pm 0.02$ & $0.17 \pm 0.01$ & 0.86 & $<10^{-9}$ & 0.34 & $2.5 \times 10^{-3}$ & 0.78 & $<10^{-9}$ & RWB \\
\hline PMN 0413-5332 & FSRQ & 763 & 11 & $0.48 \pm 0.12$ & $0.10 \pm 0.06$ & -0.26 & 0.44 & -0.49 & 0.12 & 0.97 & $9.0 \times 10^{-7}$ & RWB \\
\hline PKS 0422+004 & BL Lac & 498 & 11 & $0.42 \pm 0.09$ & $0.44 \pm 0.09$ & 0.99 & $1.5 \times 10^{-8}$ & -0.28 & 0.40 & -0.13 & 0.71 & None \\
\hline PKS 0426-380 & FSRQ & 419 & 163 & $0.35 \pm 0.02$ & $0.34 \pm 0.02$ & 0.92 & $<10^{-9}$ & -0.18 & 0.02 & 0.22 & $4.9 \times 10^{-3}$ & RWB \\
\hline NRAO 190 & FSRQ & 236 & 15 & $0.86 \pm 0.16$ & $0.77 \pm 0.14$ & 0.94 & $1.7 \times 10^{-7}$ & 0.32 & 0.24 & 0.62 & 0.01 & RWB \\
\hline PKS 0454-234 & FSRQ & 847 & 286 & $0.61 \pm 0.03$ & $0.68 \pm 0.03$ & 0.99 & $<10^{-9}$ & -0.62 & $<10^{-9}$ & -0.48 & $<10^{-9}$ & BWB \\
\hline PKS 0454-46 & FSRQ & 1193 & 47 & $0.14 \pm 0.02$ & $0.15 \pm 0.02$ & 0.21 & 0.16 & -0.61 & $5.6 \times 10^{-6}$ & 0.65 & $8.5 \times 10^{-7}$ & RWB(BWB) \\
\hline S3 0458-02 & FSRQ & 496 & 39 & $0.34 \pm 0.04$ & $0.31 \pm 0.04$ & 0.93 & $<10^{-9}$ & 0.12 & 0.45 & 0.48 & $1.9 \times 10^{-3}$ & RWB \\
\hline PKS $0502+049$ & FSRQ & 309 & 36 & $0.52 \pm 0.06$ & $0.34 \pm 0.04$ & 0.76 & $9.4 \times 10^{-8}$ & 0.01 & 0.97 & 0.66 & $1.2 \times 10^{-5}$ & RWB \\
\hline PMN 0507-6104 & FSRQ & 816 & 6 & $0.61 \pm 0.18$ & $0.66 \pm 0.19$ & 0.75 & 0.09 & -0.25 & 0.64 & 0.46 & 0.36 & None \\
\hline PKS 0528+134 & FSRQ & 2091 & 160 & $0.54 \pm 0.03$ & $0.31 \pm 0.02$ & 0.78 & $<10^{-9}$ & 0.23 & $4.1 \times 10^{-3}$ & 0.79 & $<10^{-9}$ & RWB \\
\hline PMN J0531-4827 & - & 1215 & 175 & $0.94 \pm 0.05$ & $1.01 \pm 0.05$ & 0.98 & $<10^{-9}$ & -0.34 & $3.6 \times 10^{-6}$ & -0.13 & 0.09 & BWB \\
\hline PKS 0537-441 & FSRQ & 1115 & 141 & $0.77 \pm 0.05$ & $0.66 \pm 0.04$ & 0.97 & $<10^{-9}$ & 0.16 & 0.05 & 0.40 & $1.1 \times 10^{-6}$ & RWB \\
\hline PMN J0623+3350 & - & 61 & 20 & $0.40 \pm 0.07$ & $0.54 \pm 0.09$ & 0.75 & $1.2 \times 10^{-4}$ & -0.67 & $1.2 \times 10^{-3}$ & -0.02 & 0.93 & BWB \\
\hline J0630.9-2406 & BL Lac & 827 & 18 & $0.21 \pm 0.04$ & $0.12 \pm 0.02$ & 0.37 & 0.14 & -0.26 & 0.30 & 0.81 & $5.5 \times 10^{-5}$ & RWB \\
\hline PKS 0637-75 & FSRQ & 1193 & 72 & $0.09 \pm 0.01$ & $0.07 \pm 0.01$ & 0.30 & 0.01 & -0.46 & $5.7 \times 10^{-5}$ & 0.72 & $<10^{-9}$ & RWB(BWB) \\
\hline PKS 0727-11 & FSRQ & 989 & 18 & $0.64 \pm 0.12$ & $0.88 \pm 0.15$ & 0.96 & $<10^{-9}$ & -0.86 & $4.1 \times 10^{-6}$ & -0.69 & $1.7 \times 10^{-3}$ & BWB \\
\hline PMN J0816-1311 & BL Lac & 843 & 21 & $0.22 \pm 0.03$ & $0.23 \pm 0.04$ & 0.97 & $<10^{-9}$ & -0.36 & 0.10 & -0.11 & 0.62 & None \\
\hline PKS 0818-128 & BL Lac & 826 & 20 & $0.23 \pm 0.04$ & $0.36 \pm 0.06$ & 0.53 & 0.02 & -0.76 & $1.1 \times 10^{-4}$ & 0.16 & 0.51 & BWB \\
\hline BZQ J0850-1213 & FSRQ & 1206 & 97 & $0.55 \pm 0.04$ & $0.53 \pm 0.04$ & 0.91 & $<10^{-9}$ & -0.21 & 0.04 & 0.21 & 0.04 & None \\
\hline OJ 287 & BL Lac & 2203 & 364 & $0.42 \pm 0.02$ & $0.41 \pm 0.02$ & 0.92 & $<10^{-9}$ & -0.04 & 0.49 & 0.35 & $<10^{-9}$ & RWB \\
\hline PKS 1004-217 & FSRQ & 1087 & 81 & $0.20 \pm 0.02$ & $0.14 \pm 0.01$ & -0.12 & 0.29 & -0.72 & $<10^{-9}$ & 0.77 & $<10^{-9}$ & RWB(BWB) \\
\hline PKS 1056-113 & BL Lac & 728 & 43 & $0.37 \pm 0.04$ & $0.43 \pm 0.05$ & 0.97 & $<10^{-9}$ & -0.35 & 0.02 & -0.10 & 0.52 & None \\
\hline PKS 1124-186 & FSRQ & 351 & 34 & $0.34 \pm 0.04$ & $0.34 \pm 0.04$ & 1.00 & $<10^{-9}$ & 0.54 & $9.5 \times 10^{-4}$ & 0.60 & $2.0 \times 10^{-4}$ & RWB \\
\hline PKS $1127-14$ & FSRQ & 1103 & 37 & $0.21 \pm 0.03$ & $0.17 \pm 0.02$ & -0.12 & 0.49 & -0.62 & $4.6 \times 10^{-5}$ & 0.85 & $<10^{-9}$ & RWB(BWB) \\
\hline PKS 1144-379 & FSRQ & 1115 & 128 & $0.56 \pm 0.04$ & $0.65 \pm 0.04$ & 0.93 & $<10^{-9}$ & -0.66 & $<10^{-9}$ & -0.34 & $6.9 \times 10^{-5}$ & BWB \\
\hline QSO B1212+078 & BL Lac & 942 & 9 & $0.07 \pm 0.02$ & $0.12 \pm 0.03$ & 0.47 & 0.21 & -0.78 & 0.01 & 0.18 & 0.63 & BWB \\
\hline 3 C 273 & FSRQ & 2156 & 287 & $0.06 \pm 0.00$ & $0.04 \pm 0.00$ & 0.14 & 0.02 & -0.54 & $<10^{-9}$ & 0.76 & $<10^{-9}$ & RWB(BWB) \\
\hline PKS $1244-255$ & FSRQ & 1032 & 107 & $0.55 \pm 0.04$ & $0.48 \pm 0.03$ & 0.98 & $<10^{-9}$ & 0.69 & $<10^{-9}$ & 0.81 & $<10^{-9}$ & RWB \\
\hline $3 \mathrm{C} 279$ & FSRQ & 2207 & 447 & $0.68 \pm 0.02$ & $0.72 \pm 0.02$ & 0.99 & $<10^{-9}$ & -0.46 & $<10^{-9}$ & -0.33 & $<10^{-9}$ & BWB \\
\hline PKS 1329-049 & FSRQ & 376 & 11 & $0.24 \pm 0.08$ & $0.48 \pm 0.11$ & 0.08 & 0.82 & -0.73 & 0.01 & 0.62 & 0.04 & BWB \\
\hline PKS 1335-127 & FSRQ & 1077 & 54 & $0.91 \pm 0.09$ & $0.87 \pm 0.08$ & 0.95 & $<10^{-9}$ & 0.10 & 0.47 & 0.42 & $1.6 \times 10^{-3}$ & RWB \\
\hline PKS B1406-076 & FSRQ & 2201 & 180 & $0.61 \pm 0.03$ & $0.44 \pm 0.02$ & 0.80 & $<10^{-9}$ & 0.14 & 0.06 & 0.71 & $<10^{-9}$ & RWB \\
\hline PKS B1424-418 & FSRQ & 765 & 278 & $0.71 \pm 0.03$ & $0.74 \pm 0.03$ & 0.99 & $<10^{-9}$ & -0.38 & $<10^{-9}$ & -0.26 & $1.3 \times 10^{-5}$ & BWB \\
\hline PKS 1508-05 & FSRQ & 745 & 25 & $0.20 \pm 0.03$ & $0.10 \pm 0.02$ & 0.25 & 0.22 & -0.27 & 0.20 & 0.86 & $2.7 \times 10^{-8}$ & RWB \\
\hline
\end{tabular}

of $R$ and $J$ band in blazars are comparable. Ghosh et al. (2000) suggested that it may not be correct to generalize that the amplitude of the variation in blazars is systematically larger at higher frequency. However, as can be seen in Fig. $1, F_{v a r} \mathrm{~s}$ of $J$ band are generally larger than those of $R$ band for most of FSRQs (with prob. $=0.025$ ), while $F_{v a r} \mathrm{~s}$ of $J$ band are, in general, smaller than those of $R$ band for most of BL Lacs (with prob. $=0.033$ ). Bonning et al. (2012) also found that 8 out of 9 FSRQs are more variable in $J$ band than $B$ band. As can also be seen in Fig. 1, the $F_{v a r}$ s of FSRQs seem to be larger than those of BL Lacs. Fig. 3 displays the $F_{v a r}$ distribution of FSRQs and BL Lacs in $J$ band. One can clearly see that $J$ band $F_{v a r} s$ of FSRQs are larger than those of BL Lacs. The difference is significant at a level of $5.7 \times 10^{-4}$.

We investigate the correlation between $R$ and $J$ bands. The $R$ band versus $J$ band is plotted in the left panels in Fig. 4. Linear fitting is carried out by the least square method (the solid line in the figure). In order to quantitatively search for the relation between these two bands, we calculate the correlation coefficient and estimate the significance level, and list them in Columns 7 and 8 of Table 1. From Fig. 4 
Table 1 Continued.

\begin{tabular}{|c|c|c|c|c|c|c|c|c|c|c|c|c|}
\hline Object & Class & $\begin{array}{l}\Delta T \\
\text { (day) }\end{array}$ & $N$ & $J_{F v a r}$ & $R_{\text {Fvar }}$ & $r_{J-R}$ & Prob. & $r_{C-R}$ & Prob. & $r_{C-J}$ & Prob. & Behavior \\
\hline PKS 1510-089 & FSRQ & 2207 & 405 & $0.96 \pm 0.03$ & $0.85 \pm 0.03$ & 0.96 & $<10^{-9}$ & 0.53 & $<10^{-9}$ & 0.74 & $<10^{-9}$ & RWB \\
\hline PKS 1514-241 & BL Lac & 1065 & 68 & $0.15 \pm 0.01$ & $0.15 \pm 0.01$ & 0.81 & $<10^{-9}$ & -0.27 & 0.03 & 0.35 & $3.1 \times 10^{-3}$ & RWB \\
\hline PKS $1550-242$ & - & 304 & 17 & $0.55 \pm 0.10$ & $0.57 \pm 0.10$ & 0.97 & $<10^{-9}$ & -0.23 & 0.38 & 0.00 & 1.00 & None \\
\hline PMN J1610-6649 & BL Lac & 741 & 16 & $0.19 \pm 0.03$ & $0.16 \pm 0.03$ & 0.97 & $<10^{-9}$ & 0.53 & 0.03 & 0.72 & $1.8 \times 10^{-3}$ & RWB \\
\hline PKS 1622-253 & FSRQ & 988 & 6 & $0.46 \pm 0.13$ & $0.48 \pm 0.15$ & 0.74 & 0.09 & -0.03 & 0.95 & 0.65 & 0.16 & RWB \\
\hline PKS 1622-297 & FSRQ & 2165 & 221 & $0.54 \pm 0.03$ & $0.37 \pm 0.02$ & 0.85 & $<10^{-9}$ & 0.27 & $4.9 \times 10^{-5}$ & 0.74 & $<10^{-9}$ & RWB \\
\hline $1717-5156$ & - & 455 & 133 & $0.88 \pm 0.05$ & $0.39 \pm 0.02$ & 0.86 & $<10^{-9}$ & 0.59 & $<10^{-9}$ & 0.92 & $<10^{-9}$ & RWB \\
\hline PKS 1730-130 & FSRQ & 1985 & 243 & $0.41 \pm 0.02$ & $0.32 \pm 0.01$ & 0.79 & $<10^{-9}$ & -0.05 & 0.39 & 0.57 & $<10^{-9}$ & RWB \\
\hline OT 081 & BL Lac & 379 & 14 & $0.81 \pm 0.15$ & $0.85 \pm 0.16$ & 0.99 & $<10^{-9}$ & -0.33 & 0.25 & -0.19 & 0.51 & None \\
\hline PMN J1921-1607 & BL Lac & 543 & 13 & $0.28 \pm 0.06$ & $0.23 \pm 0.05$ & 0.96 & $2.3 \times 10^{-7}$ & 0.63 & 0.02 & 0.82 & $5.6 \times 10^{-4}$ & RWB \\
\hline PKS 1954-388 & FSRQ & 1118 & 54 & $0.42 \pm 0.04$ & $0.29 \pm 0.03$ & 0.96 & $<10^{-9}$ & 0.72 & $<10^{-9}$ & 0.88 & $<10^{-9}$ & RWB \\
\hline PKS 1958-179 & FSRQ & 919 & 18 & $1.09 \pm 0.18$ & $1.17 \pm 0.20$ & 0.98 & $<10^{-9}$ & -0.59 & 0.01 & -0.42 & 0.08 & BWB \\
\hline PKS 2052-474 & FSRQ & 1553 & 176 & $0.80 \pm 0.04$ & $0.77 \pm 0.04$ & 0.96 & $<10^{-9}$ & 0.10 & 0.18 & 0.39 & $7.8 \times 10^{-8}$ & RWB \\
\hline $\begin{array}{l}\text { 2FGL J2055.4 } \\
-0023\end{array}$ & BL Lac & 752 & 11 & $0.15 \pm 0.04$ & $0.21 \pm 0.05$ & -0.29 & 0.38 & -0.83 & $1.4 \times 10^{-3}$ & 0.77 & $5.4 \times 10^{-3}$ & $\mathrm{BWB}(\mathrm{RWB})$ \\
\hline PKS 2142-75 & FSRQ & 1316 & 157 & $0.52 \pm 0.03$ & $0.17 \pm 0.01$ & 0.88 & $<10^{-9}$ & 0.77 & $<10^{-9}$ & 0.98 & $<10^{-9}$ & RWB \\
\hline PKS 2149-306 & FSRQ & 158 & 22 & $0.17 \pm 0.03$ & $0.09 \pm 0.01$ & 0.92 & $2.0 \times 10^{-9}$ & 0.72 & $1.7 \times 10^{-4}$ & 0.94 & $<10^{-9}$ & RWB \\
\hline PKS 2155-304 & BL Lac & 1940 & 363 & $0.38 \pm 0.01$ & $0.37 \pm 0.01$ & 0.99 & $<10^{-9}$ & 0.06 & 0.29 & 0.22 & $1.7 \times 10^{-5}$ & RWB \\
\hline $3 \mathrm{C} 446$ & BL Lac & 1111 & 56 & $0.34 \pm 0.04$ & $0.50 \pm 0.05$ & 0.35 & $7.3 \times 10^{-3}$ & -0.60 & $9.8 \times 10^{-7}$ & 0.53 & $2.2 \times 10^{-5}$ & $\mathrm{BWB}(\mathrm{RWB})$ \\
\hline PKS 2227-08 & FSRQ & 915 & 43 & $0.28 \pm 0.03$ & $0.51 \pm 0.06$ & 0.32 & 0.04 & -0.87 & $<10^{-9}$ & 0.18 & 0.24 & BWB \\
\hline PKS 2232-488 & FSRQ & 661 & 10 & $0.41 \pm 0.09$ & $0.26 \pm 0.06$ & 0.66 & 0.04 & 0.02 & 0.96 & 0.76 & 0.01 & RWB \\
\hline PKS 2233-148 & BL Lac & 507 & 122 & $0.76 \pm 0.05$ & $0.83 \pm 0.05$ & 1.00 & $<10^{-9}$ & -0.53 & $<10^{-9}$ & -0.45 & $2.6 \times 10^{-7}$ & BWB \\
\hline PKS 2240-260 & BL Lac & 1147 & 20 & $0.24 \pm 0.04$ & $0.22 \pm 0.04$ & 0.92 & $1.1 \times 10^{-8}$ & 0.22 & 0.35 & 0.59 & $6.6 \times 10^{-3}$ & RWB \\
\hline 3C 454.3 & FSRQ & 1985 & 505 & $0.90 \pm 0.03$ & $0.81 \pm 0.03$ & 0.99 & $<10^{-9}$ & 0.76 & $<10^{-9}$ & 0.85 & $<10^{-9}$ & RWB \\
\hline PKS 2255-282 & FSRQ & 947 & 42 & $0.57 \pm 0.06$ & $0.49 \pm 0.05$ & 0.94 & $<10^{-9}$ & 0.26 & 0.09 & 0.58 & $6.6 \times 10^{-5}$ & RWB \\
\hline 1ES 2322-409 & BL Lac & 809 & 19 & $0.20 \pm 0.03$ & $0.23 \pm 0.04$ & 0.96 & $<10^{-9}$ & -0.58 & $9.1 \times 10^{-3}$ & -0.33 & 0.17 & BWB \\
\hline PKS 2326-502 & FSRQ & 128 & 76 & $0.58 \pm 0.05$ & $0.72 \pm 0.06$ & 0.97 & $<10^{-9}$ & -0.56 & $1.8 \times 10^{-7}$ & -0.35 & $2.2 \times 10^{-3}$ & BWB \\
\hline PMN 2345-1555 & FSRQ & 790 & 167 & $0.57 \pm 0.03$ & $0.63 \pm 0.03$ & 0.98 & $<10^{-9}$ & -0.40 & $1.1 \times 10^{-7}$ & -0.19 & 0.01 & BWB \\
\hline PKS 2345-16 & FSRQ & 372 & 22 & $0.81 \pm 0.12$ & $0.79 \pm 0.12$ & -0.97 & $<10^{-9}$ & -0.99 & $<10^{-9}$ & 0.99 & $<10^{-9}$ & RWB(BWB) \\
\hline Н 2356-309 & BL Lac & 478 & 10 & $0.09 \pm 0.02$ & $0.18 \pm 0.04$ & 0.69 & 0.03 & -0.83 & $3.1 \times 10^{-3}$ & -0.16 & 0.65 & BWB \\
\hline $4 C+01.28$ & BL Lac & 27 & 8 & $0.35 \pm 0.09$ & $0.29 \pm 0.07$ & -0.95 & $3.5 \times 10^{-4}$ & -0.99 & $4.6 \times 10^{-6}$ & 0.99 & $7.2 \times 10^{-6}$ & $\mathrm{BWB}(\mathrm{RWB})$ \\
\hline
\end{tabular}

and the correlation coefficient, one can see that blazars show well-correlated variations between $R$ and $J$ bands, except for several sources showing weak or no, even negative correlations.

\section{COLOR VARIATIONS}

The color variability can reflect the spectral variability. Since these sources are highly variable, simultaneous or quasi-simultaneous observations are essential for color index calculation. The time series in the optical $R$ band and the infrared $J$ band are quasi-simultaneous and the best sampled in the SMARTS data. This provides us a chance to study in detail the spectral variations of the large sample of blazars on long time scales. $J-R$ color index is calculated pair by pair between $R$ and $J$ bands within the time interval of 15 minutes for each object. More than 90 percent of intervals are less than 5 minutes. Very few extremely strange data points have been discarded. The middle and right panels of Fig. 4 show the $J-R$ color variations with the brightness of $R$ band and $J$ band, respectively. To obtain the relation between the color index and the source brightness, we calculate the correlation coefficient $r$, and estimate the significance level. If $r>=0.2$ and the significance level $p<=0.01$, it means somewhat a good correlation between color index and magnitude. It suggests that the source becomes redder when the source becomes brighter. If $r<=-0.2$ and the significance level $p<=0.01$, it means a well negative correlation, and suggests that the source tends to be bluer when the source is brighter. The other cases mean there is no correlation between color index and source brightness. Column 9 of Table 1 lists the correlation coefficient between the color index and the magnitude of $R$ band (denoted by $r_{C-R}$ ). Its significance level is estimated and listed in Column 10. The correlation coefficient between the color index and the magnitude of $J$ band $\left(r_{C-J}\right)$ and its significance level are also calculated and listed in Columns 11 and 12. Overall, 43 sources (35 FSRQs, 7 BL Lacs and 1 unidentified object) show RWB trends and 24 sources (11 FSRQs, 11 BL Lacs and 2 unidentified objects) show BWB trends. There are 


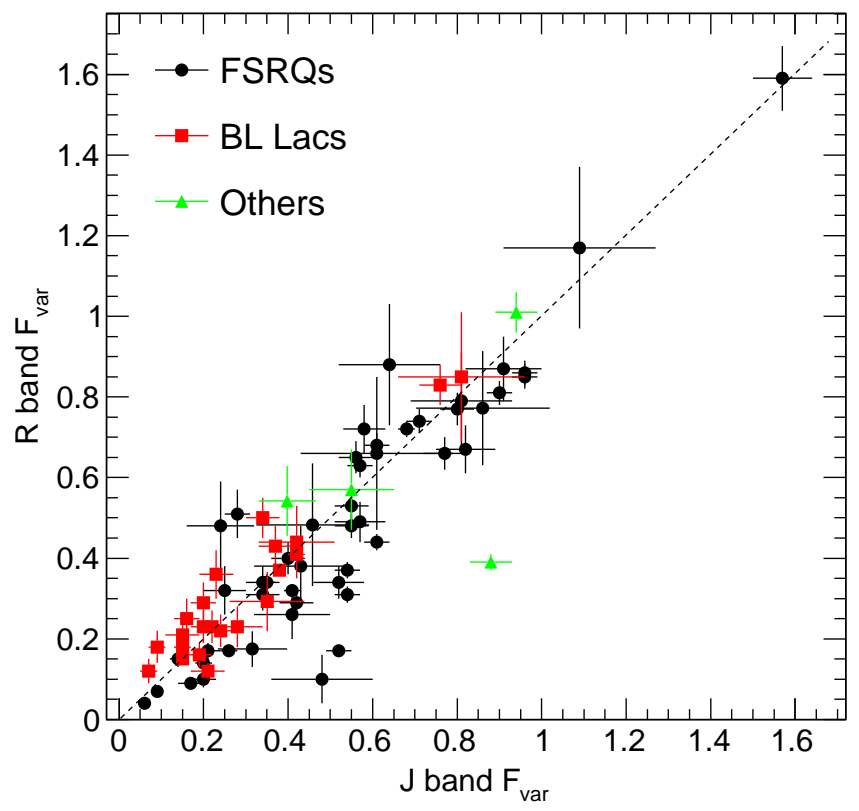

Fig. $1 F_{v a r} \mathrm{~s}$ of $R$ band versus those of $J$ band. The dots, squares and triangles represent FSRQs, BL Lacs and unidentified type objects, respectively. The dashed line means that the $F_{v a r}$ of $J$ band equal to that of $R$ band.

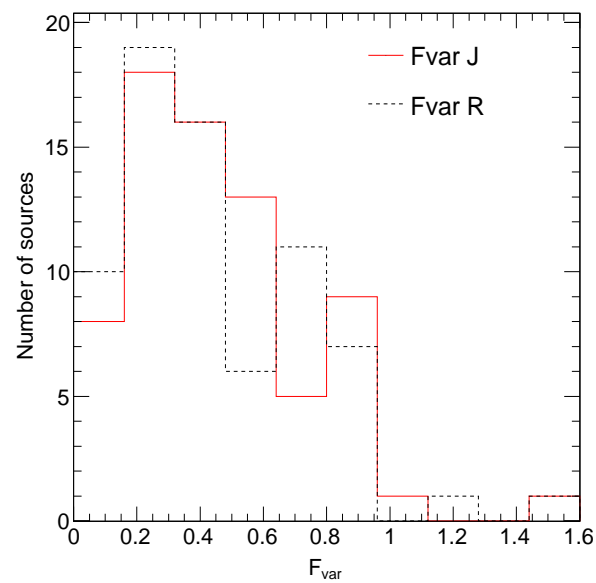

Fig. 2 Distributions of $F_{\text {vars }}$ of the optical $R$ band (the dashed line) and the infrared $J$ band (the solid line).

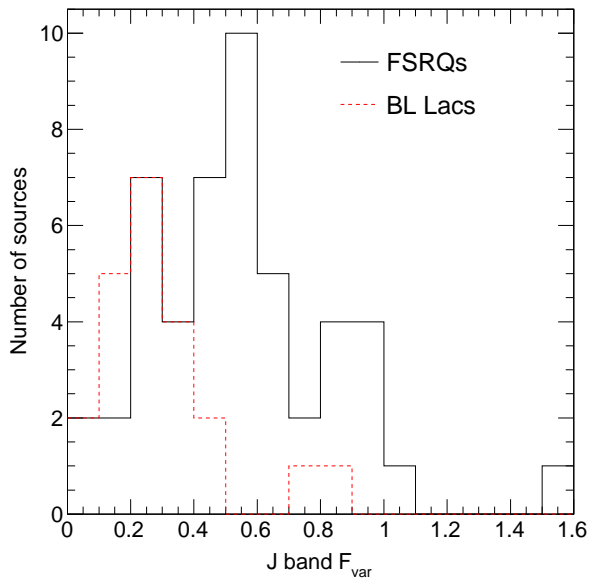

Fig. 3 Distributions of $J$ band $F_{\text {var }} s$ of FSRQs (the solid line) and BL Lacs (the dashed line).

10 sources (6 FSRQs and 4 BL Lacs) showing BWB trends in the Color $-R$ plane and opposite trends in the Color $-J$ plane. The color behaviors are listed in Column 13 of Table 1. 


\section{DISCUSSION AND CONCLUSIONS}

The BWB behavior is usually observed in BL Lac objects, which can usually be explained with shockin-jet models. This behavior maybe means the presence of two components contributing to the overall emission, a variable component with a constant and relatively blue color and an underlying red component (Fiorucci et al. 2004; Ikejiri et al. 2011). RWB behavior is generally observed in FSRQs, which can be interpreted as due to a strong contribution of a blue thermal emission from the accretion disc, which mainly affects the bluer region of the optical spectrum when the jet emission is faint (Villata et al. 2006; Rani et al. 2010; Bonning et al. 2012).

An interesting result is that 10 sources show one color behavior in one band and an opposite trend in another band. When the $R$ band (higher frequency) brightness increases, they tend to be BWB trends. However, they show RWB behavior when the source brightness increases in the $J$ band (lower frequency). This phenomena had ever clearly appeared in the case of 3C 273 (Dai et al. 2009). This may be caused by the brightness variations in the higher frequency leading those in the lower frequency. In other words, the radiation emitted at higher frequencies will emerge sooner while the maximum flux at lower frequencies will be observed later (Villata et al. 2000; Papadakis et al. 2004; Villata et al. 2009; Zhang et al. 2010b; Rani et al. 2010). Thus the variations in lower frequencies can not keep step with those in high frequencies. One would observed weak or none, even negative correlations between the two bands. Therefore, the object exhibits BWB trend in the higher frequency band, while RWB trend in the lower frequency band. We check the brightness correlations between $J$ and $R$ bands, and find that the correlations are weak or negative for these sources.

For the sources with strong brightness correlations between $R$ and $J$ bands, their color behaviors in the $R$ band are similar to those in the $J$ band, showing either BWB or RWB behavior. We investigate the relation between color behavior and $F_{\text {var }}$ difference of $R$ and $J$ bands for those objects with the absolute value of $r_{J-R}$ greater than 0.5. In Fig. 5, we plot the $r_{C-R}$ against the $F_{v a r}$ difference, $F_{v a r R}-F_{\text {var } J}$. Overall, if $F_{v a r}$ of $R$ band is greater than that of $J$ band, $r_{C-R}<0$, which means the source showing BWB trend. If $F_{v a r}$ of $R$ band is less than that of $J$ band, the source shows RWB trend $\left(r_{C-R}>0\right)$.
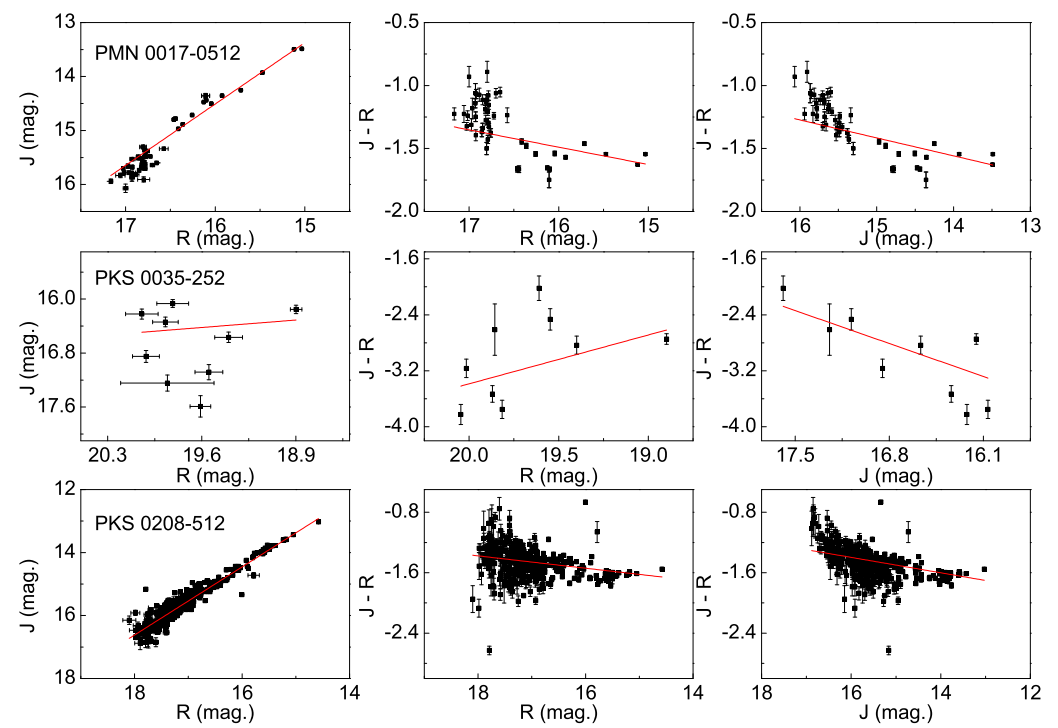

Fig. 4 Left: Relations between the $J$ and $R$ magnitudes. Middle: Color index versus the magnitude of $R$ band. Right: Color index versus the magnitude of $J$ band. The solid lines represent the best linear fitting. 


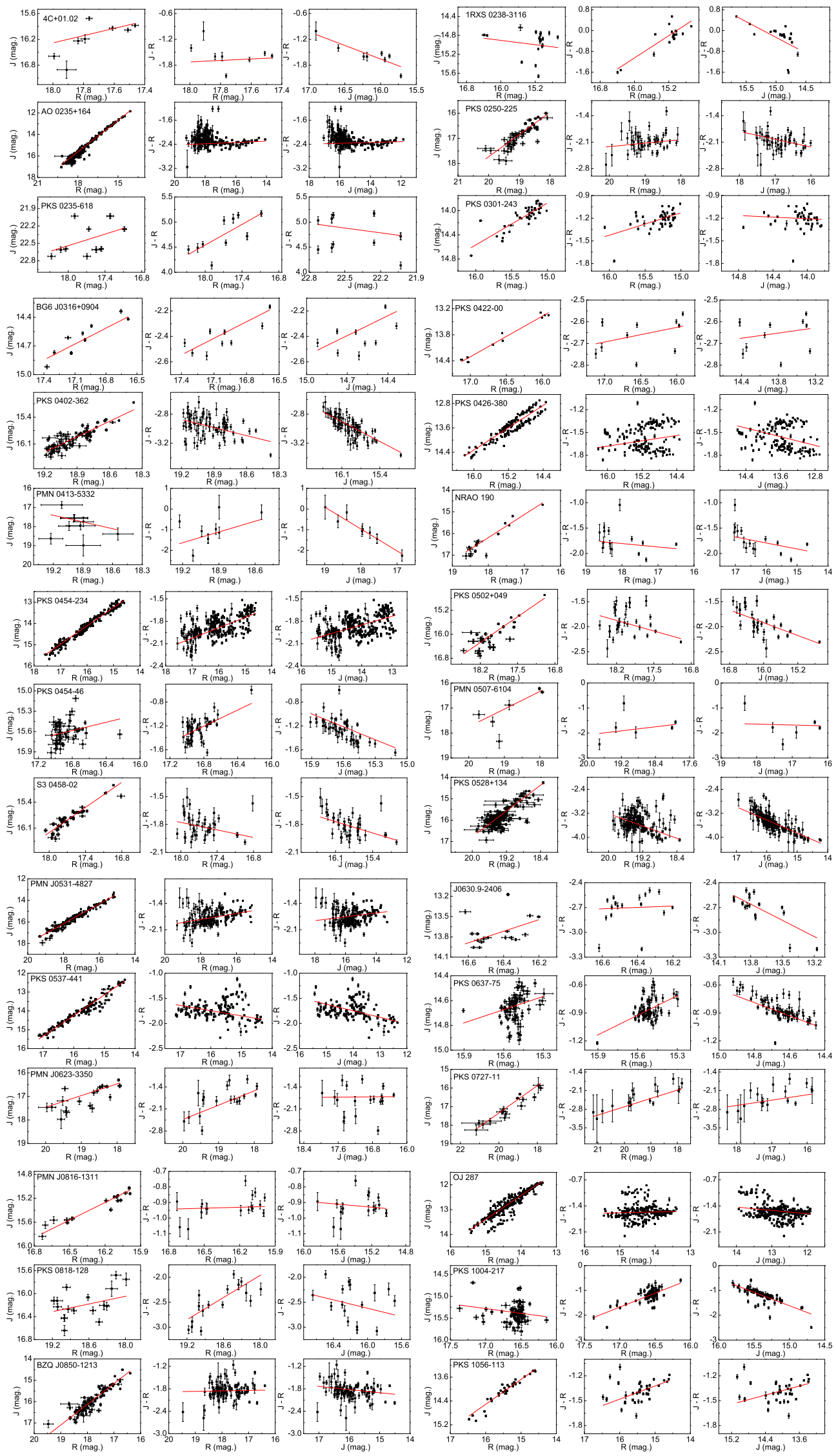

Fig. 4 Continued. 

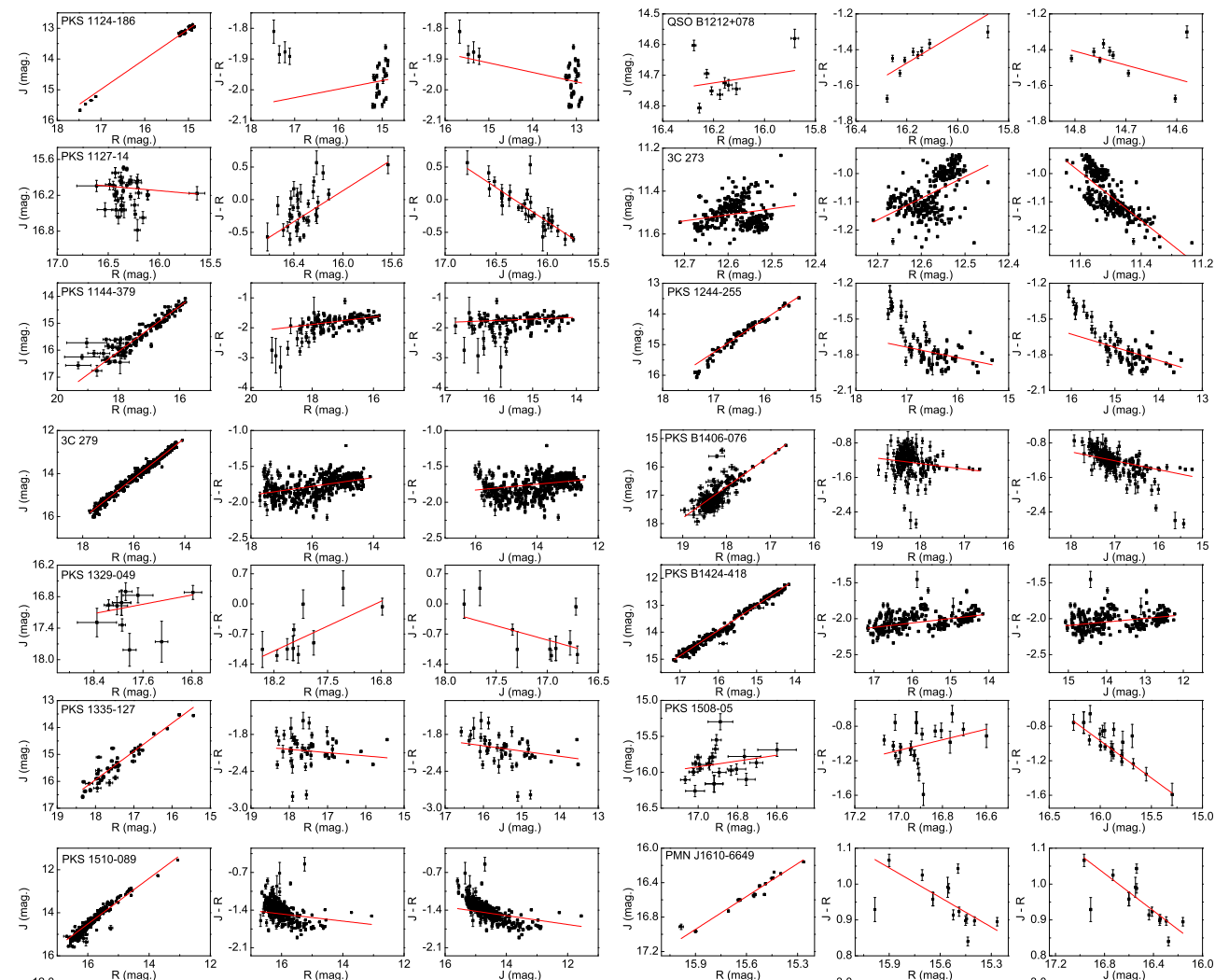

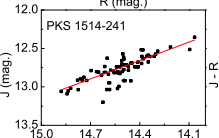
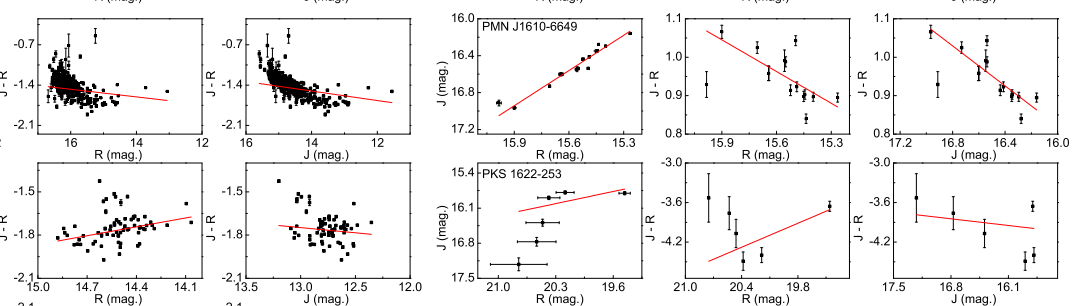

PKS 1550-242

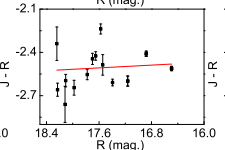

s.
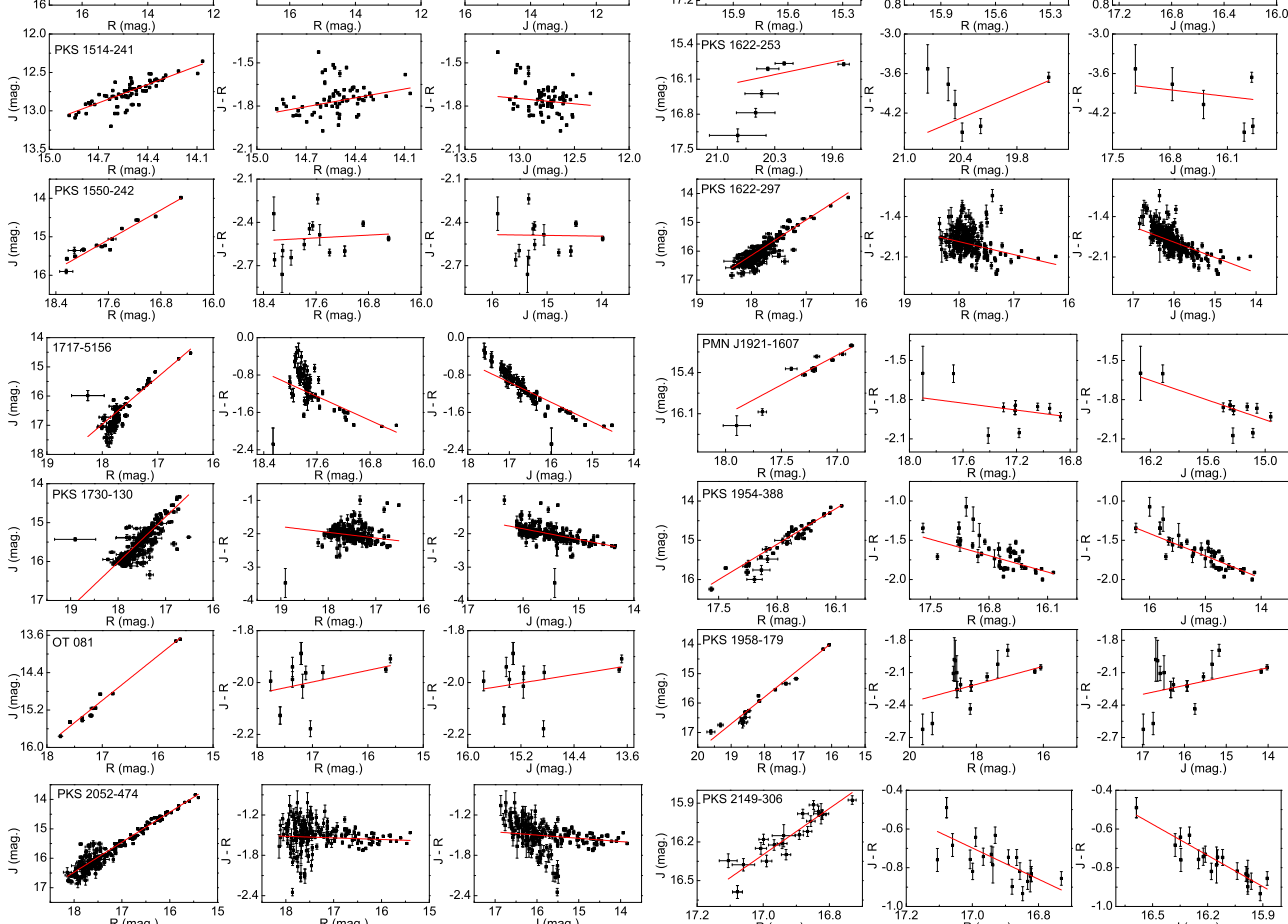

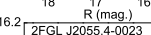
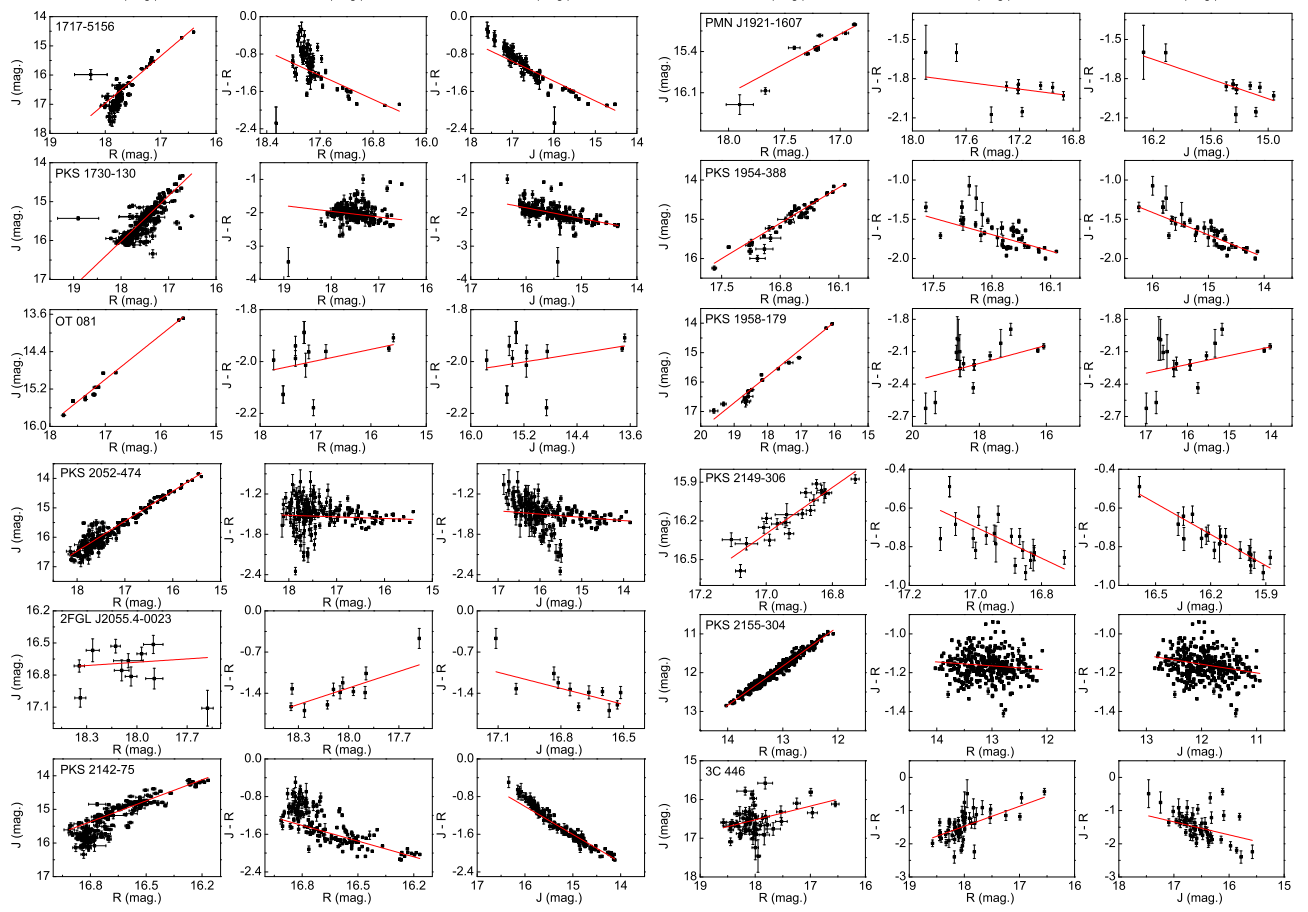

Fig. 4 Continued. 

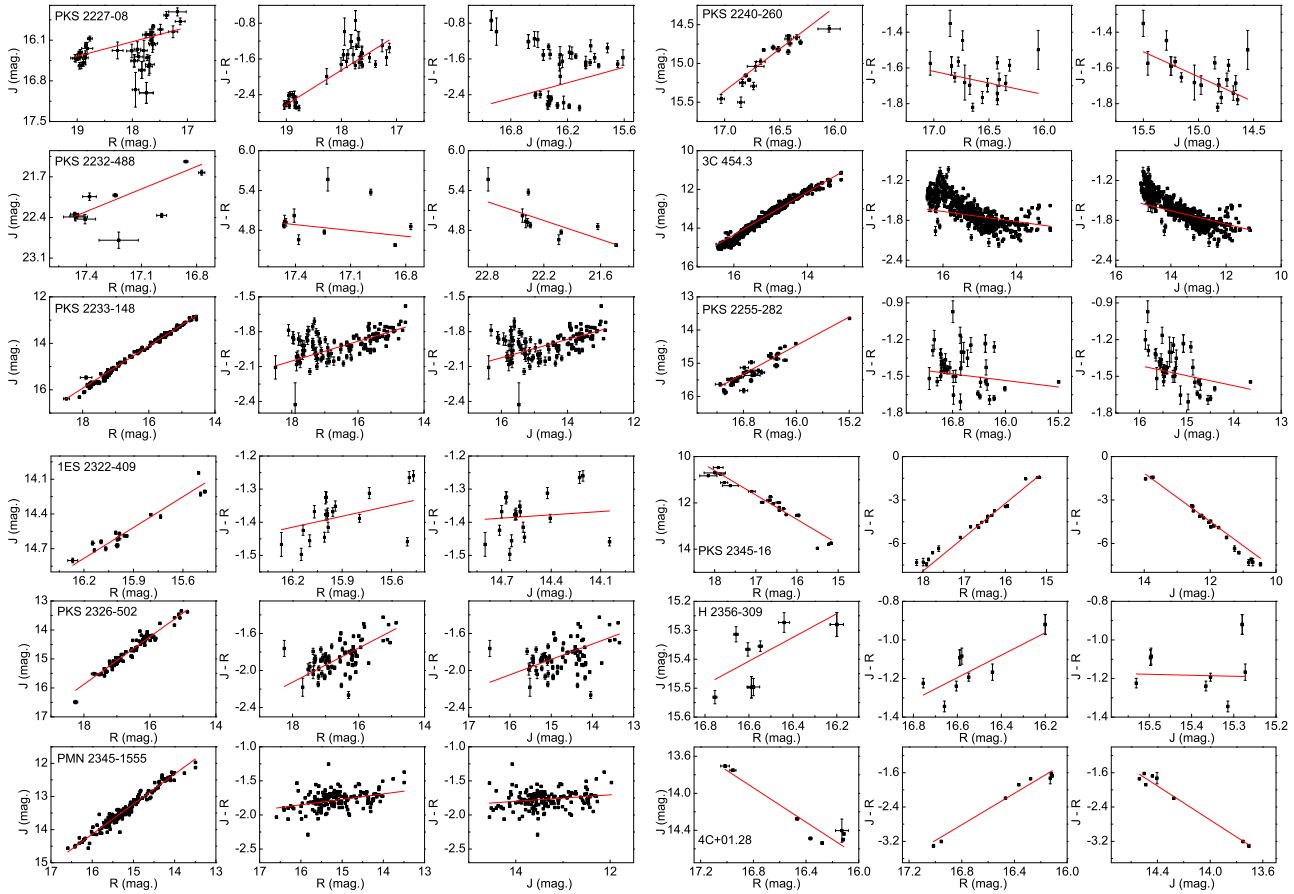

Fig. 4 Continued.

This means that the difference in variation amplitude can cause different color behaviors. For an object with the fluxes at different frequencies varying simultaneously, if the amplitude is larger at the higher frequency than at the lower one, it exhibits the BWB trend. Oppositely, the larger amplitude variations at lower frequency can result in RWB trend.

From Fig. 4, one can see that not all sources can be fitted well by a straight line. The color variation of some sources maybe change trend at a certain magnitude. Several sources show RWB in the low state, and then keep a stable trend in the high state (SWB), such as PMN 0017-0512, PKS 0208-512, PKS 1244-255, PKS 1510-089, PKS 1622-297 and 1717-5156. Some sources show RWB trend in the low state, and BWB trend in the high state, like AO 0235+164, PKS 1056-113, PKS 2233-148, PKS 2240-260 and 3C 454.3. This behavior has also been found in other literature, such as 3C 454.3, PKS 1510-089, PG 1553+113 and PKS 0537-441 in Villata et al. (2006), Ikejiri et al. (2011) and Zhang et al. (2013). Most of these sources are FSRQs. They have a common feature that they exhibit RWB trend in the faint state and then SWB or BWB trend in the bright state. This may be explained as the contribution reversal of different emission components. In the faint state, the strong contribution of thermal emission from an accretion disk results in RWB trend. While in the bright state, the non-thermal jet emission is dominant, which would display usual BWB trend, therefore balancing or exceeding the RWB trend. Thus, the source transforms its color behavior from RWB trend to SWB or BWB trend.

A few sources show two or more different strips in the Magnitude - Magnitude or Color Magnitude diagrams. PKS 0426-380 shows two clear different behaviors. In $J-R$ diagram, it seems to be a gap of two light curves with different traces. In the Color - Magnitude diagram, there is also an intersection of two curves. One exhibits BWB trend, and another exhibits RWB trend. OJ 287 indicates two parts in the color behavior, and the data points are very scattered. The color seems to be no trend in the Color $-R$ diagram, however, show a slight RWB trend in the Color $-J$ diagram. The BWB trend had been found in this source (Vagnetti, Trevese \& Nesci 2003; Fiorucci et al. 2004; Wu et al. 2006; Dai et al. 2011). A tendency to move around a circle in Color - Magnitude diagram was also found by 


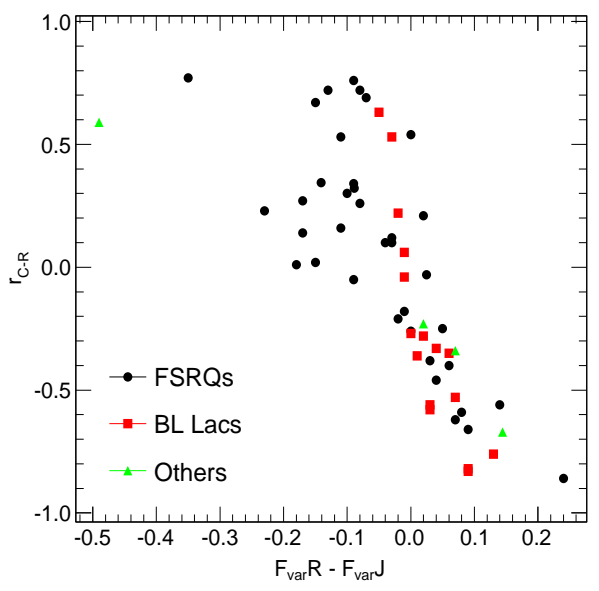

Fig. 5 Correlation coefficient $r_{C-R}$ versus the $\mathrm{F}_{v a r}$ difference of $R$ and $J$ bands.

Bonning et al. (2012) and Sandrinelli, Covino \& Treves (2014). For the 3C 273, $J-R$ plot shows two separative parts, and the Color - Magnitude plot also shows two separative parts. Two parts both have a RWB trend, and totally, Color $-J$ show RWB trend. However, in Color $-R$ plot, the combination of two part shows BWB trend. Very recently, Fan et al. (2014) also found that this source exhibited two separative parts in the plot of spectral index versus flux density. PKS 2052-474 seems to display two different behaviors in the variations both with RWB trends. PKS 2227-08 shows two separative parts, which both show RWB trend. However, the combination of two parts shows a BWB trend in $C-R$ plot. These color behaviors are puzzling, which suggest that there maybe exist more complicated and separative emission components in these sources.

In conclusion, the color variability has been investigated with a large sample of blazars in optical and infrared region. On the whole, 46 out of 49 FSRQs show color variations with RWB trends (35 FSRQs) or BWB trends (11 FSRQs), and 18 out of 22 BL Lacs show RWB trends (7 BL Lacs) or BWB trends (11 BL Lacs). Among them, 10 blazars follow RWB trend in the faint state and then SWB or BWB trend in the bright state.

Acknowledgements We express our thanks to the referee for great helps. This work has been supported by the National Natural Science Foundation of China (Grant No. 11273008), and partly by the Foundation for Young Talents in College of Anhui Province (Grant No. 2012SQRL116) . This paper has made use of up-to-date SMARTS optical/near-infrared light curves that are available at www.astro.yale.edu/smarts/glast/home.php.

\section{References}

Arévalo P., Uttley P., Kaspi S., Breedt E., Lira P., \& McHardy I. M., 2008, MNRAS, 389, 1479

Bonning E., Urry C. M., Bailyn C., et al., 2012, ApJ, 756, 13

Carini M. T., Miller H. R., Noble J. C., \& Goodrich B. D., 1992, AJ, 104, 15

Chatterjee R., Bailyn C. D., Bonning E. W., et al., 2012, ApJ, 749, 191

Ciprini S., Takalo L. O., Tosti G., et al., 2007, A\&A, 467, 465

D’Ammando F., Antolini E., Tosti G., et al., 2013, MNRAS, 431, 2481

Dai B. Z., Li X. H., Liu Z. M., et al., 2009, MNRAS, 392, 1181

Dai Y., Wu J., Zhu Z. H., Zhou X., \& Ma J., 2011, AJ, 141, 65

Fan J. H., \& Lin R. G., 2000, ApJ, 537, 101

Fan J. H., Kurtanidze O., Liu Y., Richter G. M., Chanishvili R., \& Yuan Y. H., 2014, ApJS, 213,26

Fiorucci M., Ciprini S., \& Tosti G., 2004, A\&A, 419, 25 
Gaur H., Gupta A. C., Strigachev A., et al., 2012, MNRAS, 425, 3002

Ghosh K. K., Ramsey B. D., Sadun A. C., \& Soundararajaperumal S., 2000, ApJS, 127, 11

Gu M. F., Lee C. U., Pak S., Yim H. S., \& Fletcher A. B., 2006, A\&A, 450, 39

Gu M. F., \& Ai Y. L., 2011, A\&A, 528, 95

Hu S. M., Wu J. H., Zhao G., \& Zhou X., 2006, MNRAS, 373, 209

Ikejiri Y., Uemura M., Sasada M., et al., 2011, PASJ, 63, 639

Papadakis I. E., Boumis P., Samaritakis V., \& Papamastorakis J., 2003, A\&A, 397, 565

Papadakis I. E., Samaritakis V., Boumis P., \& Papamastorakis J., 2004, A\&A, 426, 437

Raiteri C. M., Villata M., Tosti G., et al., 2003, A\&A, 402, 151

Rani B., Gupta A. C., Strigachev A., et al., 2010, MNRAS, 404, 1992

Sandrinelli A., Covino S., \& Treves A., 2014, A\&A, 562, A79

Vagnetti F., Trevese D., \& Nesci R., 2003, ApJ, 590, 123

Vaughan S., Edelson R., Warwick R. S., \& Uttley P., 2003, MNRAS, 345, 1271

Villata M., Mattox J. R., Massaro E., et al., 2000, A\&A, 363, 108

Villata M., Raiteri C. M., Balonek T. J., et al., 2006, A\&A, 453, 817

Villata M., Raiteri C. M., Larionov V. M., et al., 2009, A\&A, 501, 455

Wu J., Zhou X., Ma J., \& Jiang Z., 2011, MNRAS, 418, 1640

Wu J., Zhou X., Wu X. B., et al., 2006, AJ, 132, 1256

Zhang B. K., Dai B. Z., Zhang L., \& Cao Z., 2010a, RAA, 10, 653

Zhang B. K., Dai B. Z., Zhang L., Liu J. L., \& Cao Z., 2010b, PASA, 27, 296

Zhang B. K., Wang S., Zhao X. Y., Dai B. Z., \& Zha M., 2013, MNRAS, 428, 3630

Zhang B. K., Zhao X. Y., Wang C. X., \& Dai B. Z., 2014, RAA, 14, 933 